\title{
Accurate airway centerline extraction based on topological thinning using graph-theoretic analysis
}

\author{
Zijian Bian ${ }^{\mathrm{a}, \mathrm{b}}$, Wenjun Tan ${ }^{\mathrm{b}, \mathrm{c},}$, Jinzhu Yang ${ }^{\mathrm{b}, \mathrm{c}}$, Jiren Liu ${ }^{\mathrm{a}}$ and Dazhe Zhao ${ }^{\mathrm{b}, \mathrm{c}}$ \\ ${ }^{a}$ Sino-Dutch Biomedical and Information Engineering School, Northeastern University, No. 3-11, \\ Wenhua Road, Heping District, Shenyang, P.R. China \\ ${ }^{b}$ Key Laboratory of Medical Image Computing of Ministry of Education, Northeastern University, No. \\ 3-11, Wenhua Road, Heping District, Shenyang, P.R. China \\ ${ }^{c}$ College of Information Science and Engineering, Northeastern University, No. 3-11, Wenhua Road, \\ Heping District, Shenyang, P.R. China
}

\begin{abstract}
The quantitative analysis of the airway tree is of critical importance in the CT-based diagnosis and treatment of popular pulmonary diseases. The extraction of airway centerline is a precursor to identify airway hierarchical structure, measure geometrical parameters, and guide visualized detection. Traditional methods suffer from extra branches and circles due to incomplete segmentation results, which induce false analysis in applications. This paper proposed an automatic and robust centerline extraction method for airway tree. First, the centerline is located based on the topological thinning method; border voxels are deleted symmetrically to preserve topological and geometrical properties iteratively. Second, the structural information is generated using graph-theoretic analysis. Then inaccurate circles are removed with a distance weighting strategy, and extra branches are pruned according to clinical anatomic knowledge. The centerline region without false appendices is eventually determined after the described phases. Experimental results show that the proposed method identifies more than $96 \%$ branches and keep consistency across different cases and achieves superior circle-free structure and centrality.
\end{abstract}

Keywords: Airway tree, centerline extraction, topological thinning, graph-theoretic analysis

\section{Introduction}

The interpretation of thoracic airway tree in high-resolution CT images is urged for the early diagnosis and treatment of pulmonary diseases [1]. Such work may be considerably regularized if the airway is simplified to a rooted tree shaped structure with a series of vertices and connected paths. This structure, defined as centerline, is the most typical quantity to represent airway's topological and geometrical information. The research spreads a variety of extended applications. Hierarchical pulmonary spatial divisions, e.g. lobes and segments, could be obtained consecutively under the one-to-one cor-

\footnotetext{
${ }^{*}$ Corresponding author: Wenjun Tan, Key Laboratory of Medical Image Computing of Ministry of Education, Northeastern University, No. 3-11, Wenhua Road, Heping District, Shenyang, P.R. China. Tel.: +86 130792 23202; Fax: +86 024836 63446; E-mail: tanwenjun@mail.neu.edu.cn.
}

0959-2989/14/\$27.50 @ 2014 - IOS Press and the authors. 
respondence between airway branches and lung regions. The airway furcate nodes are commonly selected as the landmarks during point-based deformable registration of longitudinal chest CT image scans [2]. A content of pulmonary properties, such as radius, wall thickness, volume and density, offers objective and rigorous assessment of the nature, severity and distribution of respiratory disorders.

Significant attention has been paid to the centerline extraction process because of its importance in pulmonology studies. Several extraction methods have been investigated to generate complete airway branches and guarantee the centrality. One frequently used method is topological thinning $[3,4]$. Border voxels are symmetrically peeled conforming to topology principles through iteration until no voxel reduction occurs. Another strategy applies a combination of distance maps on the input airway data $[5,6]$. Each segmented voxel is computed according to their relative positions from airway root and surface, forming distance field of source and boundary (DFS and DFB). Hence, centerline branches are identified from the largest DFS valued voxels (end) back to the root with local maximum DFB valued ones connected. Additionally, there is an automatic one-step approach called fast marching, which can produce centerline simultaneously with the segmentation phase [7]. The segmentation is assumed to be a front-propagation experience: airway region consecutively merges when front propagates and the marching front centers are naturally determined as centerline points.

Unfortunately, the previous work sometimes fails in an accurate version. The phenomenon arises with the high irregularity of incomplete segmentation results. The image noise and partial volume effect decrease the CT visibility of tubular walls separating airway lumen and pulmonary parenchyma. Hence, the biomedical community has to impose strict criteria to avoid small bronchi leakage into surrounded lung regions [8]. Such criteria are confided in tree shape-preserving, but they induce surface roughness and intraluminal cavities. As a result, the generated centerline is appended with extra branches and unexpected circles [9]. The bronchi would be redundantly counted during clinical analysis. Additionally, the distance-based method is rather sensitive to the distance computation rules. Some approximation strategies are adopted to reduce computation complexity of the entire airway data but the produced centerline region deviates from the central position. The evaluated radius of small bronchi substantially decreases, resulting in false quantitative measurements.

This paper provides a new automatic and robust centerline extraction method for segmented airway tree. The work is implemented in two main phases. The centerline structure is initially generated using topological thinning. Then the structure is refined based on graph-theoretic analysis. There are several distinctions between the method and previous ones. First, instead of adopting the adjacency tree, the method achieved connected component computation with a region-growing-based voxel labeling. Second, the initially extracted region is weighted, and the path searching is performed to remove unwanted circles. Finally, extra centerline branches are pruned according to thoracic anatomy. The three points contribute to more accurate and consistent results even the segmentation is incomplete. The paper is organized as follows. Section II proposes detailed centerline extraction and refinement method. Section III offers experimental results and discussion. Section IV contains the conclusion.

\section{Methodologies}

\subsection{Topological thinning}

Thinning is a commonly used method to generate a one-voxel wide centerline region directly with exact centrality. Border points whose deletion do not induce any topological property change, also known as simple points, are peeled iteratively. Such properties include connected component, hole and 
cavity. Further definitions can be seen in [10]. Typically, Euler characteristic is applied relating the number of those three properties. For a binary volume R, its Euler characteristic $\mathrm{E}$ is defined as:

$$
\mathrm{E}(\mathrm{R})=\sum \sigma(\mathrm{R})-\sum \tau(\mathrm{R})+\sum \kappa(\mathrm{R})
$$

Where $\sigma, \tau, \kappa$ are connected component, hole and cavity. Euler characteristic provides both global and local versions topologically: the Euler characteristic of the entire image is the sum of those of its subsets. Hence, the voxel reduction can be implemented successively according to voxel configuration in the $3 \times 3 \times 3$ sized neighbor cube $\mathrm{N}^{3}$ of individual border point and then sub-decomposed into eight $2 \times 2 \times 2$ sized octants $\mathrm{N}^{2}$. The Euler characteristic of voxel $p$ 's octant configuration is computed as:

$$
E\left[N^{2}(p)\right]=v\left[N^{2}(p)\right] / 8-e\left[N^{2}(p)\right] / 4+f\left[N^{2}(p)\right] / 2-\operatorname{oct}\left[N^{2}(p)\right]
$$

Where $v, e, f$, oct are the amounts of vertices, edges, faces and octants consisted of airway voxels, respectively. There are totally $2^{7}=128$ configurations in an octant, which are stored in a preserving table. Further details can be found in [10]. The Euler invariance can be then calculated according to the characteristic change after the removal of a given border point.

Besides, since there is no cavity newly generated during the deletion of border points, the Euler characteristics and connected components are enough to represent the topology-preserving. Instead of traditional adjacency tree process [10], the change of connected components in this paper is identified using a voxel labeling method. Airway voxels in $\mathrm{N}^{3}(p)$ are first counted. Then one of those airway voxels, $q_{0}$, is selected to be the seed point; the following voxels are consecutively labeled in a region growing way in order to obtain a connected component $\sigma\left(q_{0}\right)$ as Eq. (1). The connected components conserve only when the amount of labeled voxels in $\sigma\left(q_{0}\right)$ achieves the initial count of in $\mathrm{N}^{3}(p)$.

$$
q_{k+1} \neq p \wedge q_{k+1} \in R \wedge q_{k+1} \in N^{3}(p) \wedge q_{k+1} \in N^{3}\left(q_{k}\right) \wedge q_{k+1} \notin\left\{O\left(q_{0}\right)\right\} \Rightarrow q_{k+1} \in\left\{\sigma\left(q_{0}\right)\right\}
$$

Under the two property invariance conditions, border points are peeled layer-by-layer in an iterative way. Airway centerline eventually remains as no point is deleted anymore.

\subsection{Bronchial refinement}

Unfortunately, the former topological thinning procedure is rather sensitive to coarse boundary and intraluminal cavities due to the incomplete airway segmentation. Consequently, the formerly centered structure suffers some undesirable appendices, specifically, circles and extra branches. The situation contradicts with clinical thoracic anatomy. The airway has been confirmed to be a tree shaped organ whose trunk is the main trachea, and individual parent-branch hierarchically split up into a pair of child-branches with similar geometrical properties. This general tendency propagates across lung, lobe, and segment airway to small bronchi [11]. Hence, in the graph-theoretic sense, the airway centerline can be regarded as a circle-free tree: the vertex of the main trachea is the root; the $N$ furcation points and terminals are graph nodes; the $\mathrm{N}-1$ airway branches are edges. 
Several graph-theoretic processes enable further correction in order to guarantee a more accurate version. A set of subsequent bronchial refinement steps are performed using these methods, including the circle component removal and the false branch pruning.

First, the circle-removal step is implemented on the thinned region. A source distance transformation is used to produce a weighted volumetric data whose root is weighted 0 , following the rules:

$$
W(q)=\min [W(p)+D \cdot\|q, p\|], q \in R \wedge q \in N^{3}(p)-\{p\}
$$

Where $W$ denotes the weighted source distance of centerline point, $D$ is the normalized coefficient, \|\| is the Euler distance between two points. The root's weight is 0 . The transform initializes at the root and iterates in a depth-first way until weighted change happens nowhere across the input data. Thus, all the terminals of the airway tree can be found where centerline points with local-largest distance are located. The Dijkstra's algorithm is then utilized to search single-source shortest paths over the thinned region. There exists a pair of paths corresponds to a given terminal in the circle, but only the minimum weighted one is left. Center points are consecutively lined under topological connectivity and converge at the root, producing a circle-free structure.

After the circle-removal step, branch pruning is continued to exclude undesirable branches from the remaining tree-like data according to geometric information, specifically, branch length and intersection angles. This step can be divided into two parts. The centerline topological structure begins to be identified with a breadth-first search algorithm. Each branch is stored with its origin, terminal, length, member point locations. While the noise volume is limited, its corresponding branch's length is accordingly short. Thus, a terminal branch is believed to be a false candidate if it is shorter than a length threshold. In usual airway organ, the intersections between parent and child branches are similarly obtuse angles [12]. Since the airway branch is cylinder shaped, its centerline is approximately linear. Hence, the centerline branches are fitted using a least square method whose direction vector $v$ is:

$$
\boldsymbol{v}=\left(\frac{n \sum_{i=1}^{n} x_{i}^{2}-\left(\sum_{i=1}^{n} x_{i}\right)^{2}}{n \sum_{i=1}^{n} x_{i} z_{i}-\sum_{i=1}^{n} x_{i} \sum_{i=1}^{n} z_{i}}, \frac{n \sum_{i=1}^{n} y_{i}^{2}-\left(\sum_{i=1}^{n} y_{i}\right)^{2}}{n \sum_{i=1}^{n} y_{i} z_{i}-\sum_{i=1}^{n} y_{i} \sum_{i=1}^{n} z_{i}}, 1\right)
$$

Where $n$ is the branch length, $\left(x_{i}, y_{i}, z_{i}\right)$ denotes the three-dimensional coordinates of a center point belonging to the fitted branch. All false candidates are re-checked according to the intersections with their parent-branches, and branches in the cases of acute or right angles are finally pruned.

\section{Experiments}

\subsection{Data and quantitative indices}

The proposed method was tested on actual segmented airway data sets with a PC workstation with 2.30-GHz CPU (4-core) and 4-GB RAM. The segmentation was performed on nine cases segmented by a multi-scale three-dimensional region growing method described in [13]. The method can extract branches on sub-segmental level completely and avoid bronchial segmentation leakage. In most cases, 
Table 1

Image information of segmented airway scans

\begin{tabular}{llllllllll}
\hline & Case 1 & Case 2 & Case 3 & Case 4 & Case 5 & Case 6 & Case 7 & Case 8 & Case 9 \\
\hline Branch & 75 & 57 & 25 & 101 & 71 & 155 & 69 & 63 & 77 \\
Volume voxels & 261353 & 270259 & 96076 & 352770 & 225451 & 543902 & 302438 & 269814 & 327877 \\
Surface voxels & 29416 & 30947 & 10273 & 41096 & 24757 & 62838 & 33962 & 30479 & 35804 \\
\hline
\end{tabular}

there were several clusters of surface bump noise and numerous intraluminal cavities induced by the incomplete segmentation. However, it is quite difficult to recognize and correct all of them.

The test cases range from patients with severe lung disease to health volunteers across full inspiration and full expiration condition. The first three cases are clinical data provided by the cooperated hospital, and the last six cases are from public challenge data provided by EXACT09 [14]. Table 1 lists the image information of the nine input data sets. Several indices were applied to identify airway tree information: the generated branch amount, believed to be an important structural standard, was inspected and counted visually by an experienced pulmonologist; the volume and surface area represented international geometrical features were obtained from the binary segmented voxels.

\subsection{Validation scheme}

Validation of the centerline extraction would ideally be based on ground truth manually determined by clinical pulmonologists. Unfortunately, this task is not feasible due to the extreme labor intensity in the manual work. Alternatively, a validation scheme was developed to test the method in three phases.

First, the proposed method was performed on each case, as well as traditional thinning-based method in [4] and distance-based method in [6]. The breadth-first tree traversal algorithm described in Section II was applied in order to verify the topology-preserving property. The generated nodes and edges were counted to indicate extracted branches and undesirable appendices. A radius-based reproduction was implemented to prove geometric-preserving as Eq. (6). The radius of one centerline point is defined as the minimum Euler distance from segmented boundary; morphology dilation was then utilized at each centerline point according to its radius to reproduce airway shaped binary image scans.

$$
R E P(R)=\bigcup\left[\left\{p_{i}\right\} \oplus\left\{q\left\|q, p_{i}\right\| \leq \min \left(\left\|b, p_{i}\right\|\right)\right\}\right], b \in R \wedge N^{3}(b) \cap \bar{R} \neq \varnothing
$$

Second, all the three methods were tested repeatedly with multiple pruning thresholds on each case. The same algorithm above was used to determine the airway topological properties.

Third, a two-dimensional morphology hole filling was applied to eliminate intraluminal cavities [15]. The three methods were run again on the filled airway trees as well as the breadth-first traversal and data reproduction. Each extraction method was compared based on the difference with the produced centerlines and the original segmented airway in the first phase. Both the second and third step aimed to evaluate the methods' robustness performance in extra branch and circle removal processes.

\subsection{Results and discussion}

Figure 1 shows the experimental airway cases and their centerline results extracted by the proposed method, traditional thinning-based method and distance-based method respectively. On average, 20-30 
seconds are required for the nine experimental cases. Detailed indices obtained by the tree traversal and percentage results compared with the original segmented airway data are presented in Table 2.

In the cases of actual segmented airway tree, it can be seen a high matching rate for the proposed method. The matching should be further explained in two aspects. From the topological perspective, the proposed method achieved $96.3 \pm 1.9 \%$ extracted segments, and circle component was never found according to the graph-theoretic principles described relationships between tree nodes and branches in Section II. From the geometrical perspective, the reproduced airway data remained $94.9 \pm 1.7 \%$ in volume and $95.1 \pm 1.7 \%$ in surface area compared with the original data. The mean values and standard deviations imply considerable centrality of the centerline regions.

Traditional thinning-based method identified similar geometrical results. However, it failed in the topological property due to the over-extracted branches and remarkable difference across data sets. Nodes and branches determined by the thinning-based method indicated a number of extra configurations of all the nine experimental cases. This situation became especially notable in Case 4, whose false branch and circle numbers were 18 and 4. Figure 2(a) is an enlarged result version.

Although the distance-based method avoided centerline circles, even more unwanted branches and more inconsistency were recognized per case in comparison with the thinning-based method. It should be pointed out that the distance-based method revealed quite low performance in the reproduction experiment. The predicted radius abnormally decreased as centerline close to airway boundary and a variety of voxels in Case 1 was missed. The phenomenon can be seen in Figure 2(b).

The proposed method deletes voxels in a symmetrical way as well as traditional thinning-based method, which ensures the centered location of the final centerline results. Nevertheless, since the distance-based method adopts an approximate integer weighting rules to replace the decimals in Euler distance computing, its extraction result is mostly influenced by the choice of approximation. The 3-4-5 distance is typically used, but the extracted region suffered deviation of local-maximum weight voxel which determined the overall centrality. In contrast, the proposed method applies the map on only the region where the centerline located possibly to reduce computation complexity, which allowed easy and stable implementation for the time-consuming and random weighting iteration.

Table 2

Quantitative indices of extracted centerlines

\begin{tabular}{|c|c|c|c|c|c|c|c|c|c|c|c|}
\hline \multirow{2}{*}{ Methods } & \multirow{2}{*}{ Indices } & \multicolumn{9}{|l|}{ Case } & \multirow{2}{*}{ Mean \pm Stdev } \\
\hline & & 1 & 2 & 3 & 4 & 5 & 6 & 7 & 8 & 9 & \\
\hline \multirow{5}{*}{$\begin{array}{l}\text { The pro- } \\
\text { posed } \\
\text { method }\end{array}$} & Node & 74 & 56 & 24 & 98 & 70 & 154 & 68 & 62 & 74 & \\
\hline & Branch & 73 & 55 & 23 & 97 & 69 & 153 & 67 & 61 & 73 & \\
\hline & Branch (\%) & 97.3 & 96.5 & 92.0 & 96.0 & 97.2 & 98.7 & 97.1 & 96.8 & 94.8 & $96.3 \pm 1.9$ \\
\hline & Volume (\%) & 92.5 & 93.7 & 96.6 & 97.0 & 95.5 & 93.1 & 96.7 & 94.2 & 94.6 & $94.9 \pm 1.7$ \\
\hline & Surface $(\%)$ & 92.7 & 94.1 & 96.8 & 97.2 & 95.9 & 93.2 & 96.9 & 94.5 & 94.9 & $95.1 \pm 1.7$ \\
\hline \multirow{5}{*}{$\begin{array}{l}\text { Thin- } \\
\text { ning-based } \\
\text { method }\end{array}$} & Node & 73 & 62 & 34 & 112 & 70 & 152 & 68 & 62 & 82 & \\
\hline & Branch & 74 & 64 & 35 & 115 & 70 & 155 & 69 & 63 & 83 & \\
\hline & Branch (\%) & 98.7 & 112.3 & 140.0 & 113.9 & 98.6 & 100.0 & 100.0 & 100.0 & 107.8 & $107.9 \pm 13.4$ \\
\hline & Volume (\%) & 92.6 & 93.8 & 97.2 & 97.7 & 95.6 & 93.4 & 96.8 & 94.5 & 94.6 & $95.1 \pm 1.8$ \\
\hline & Surface $(\%)$ & 92.7 & 94.2 & 97.3 & 97.9 & 96.1 & 94.2 & 97.4 & 94.7 & 94.5 & $95.4 \pm 1.8$ \\
\hline \multirow{5}{*}{$\begin{array}{l}\text { Dis- } \\
\text { tance-based } \\
\text { method }\end{array}$} & Node & 84 & 72 & 38 & 108 & 74 & 152 & 78 & 54 & 74 & \\
\hline & Branch & 83 & 71 & 37 & 107 & 73 & 151 & 77 & 53 & 73 & \\
\hline & Branch (\%) & 110.7 & 124.6 & 148.0 & 105.9 & 102.8 & 97.4 & 111.6 & 84.1 & 94.8 & $108.9 \pm 18.6$ \\
\hline & Volume (\%) & 82.8 & 95.4 & 96.4 & 98.7 & 94.6 & 93.6 & 85.3 & 93.8 & 94.7 & $92.8 \pm 5.2$ \\
\hline & Surface $(\%)$ & 80.5 & 94.2 & 97.1 & 98.6 & 94.5 & 93.7 & 85.5 & 93.2 & 94.0 & $92.4 \pm 5.7$ \\
\hline
\end{tabular}




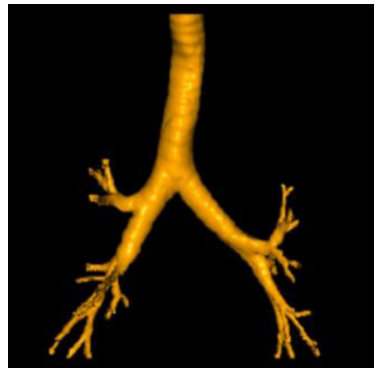

(a1)

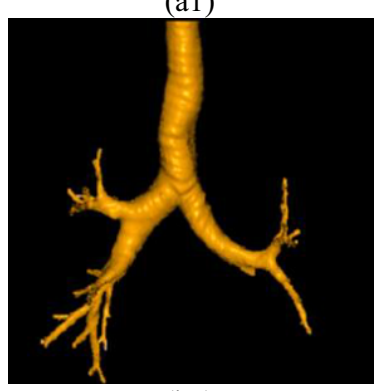

(b1)

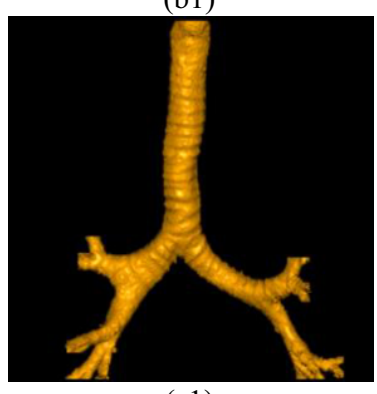

(c1)

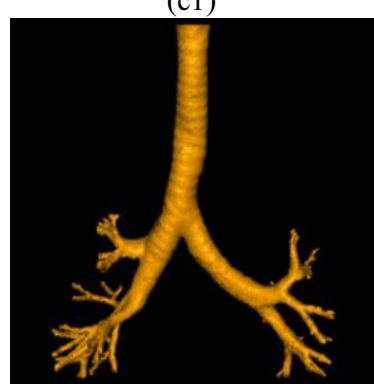

(d1)

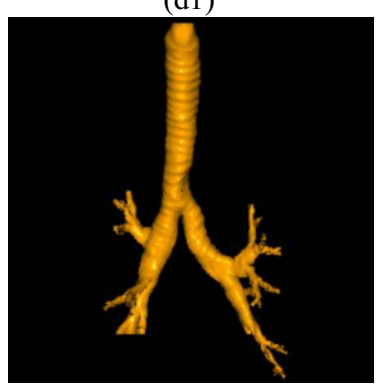

(e1)

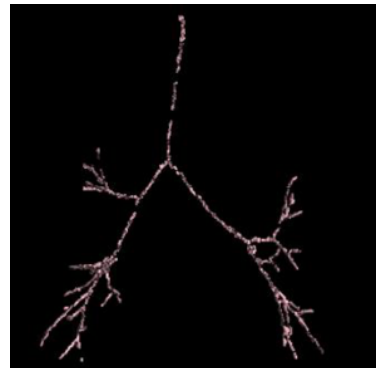

(a2)

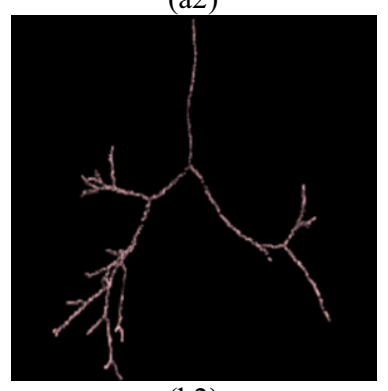

(b2)

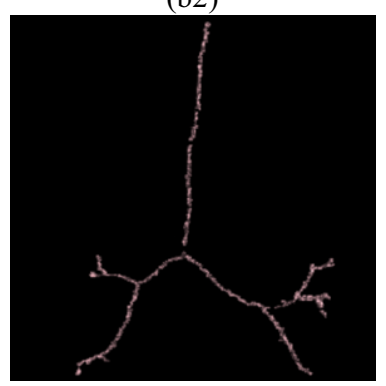

(c2)

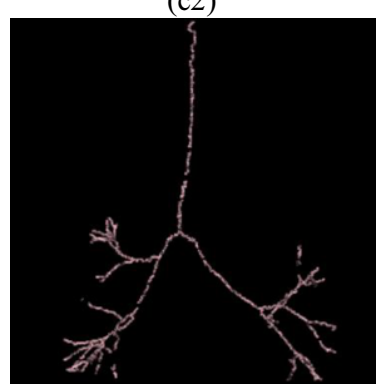

(d2)

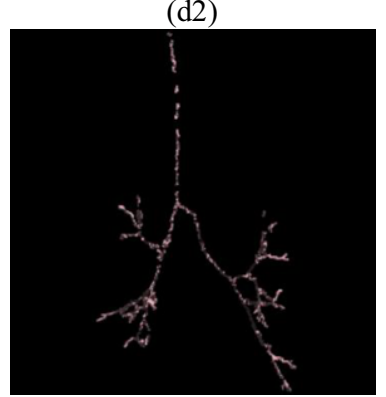

(e2)

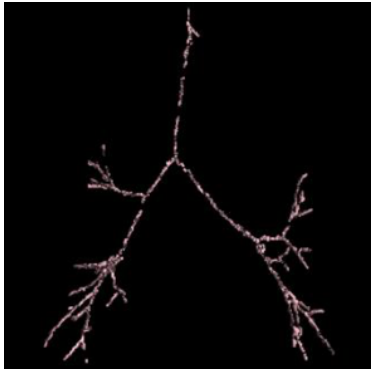

(a3)

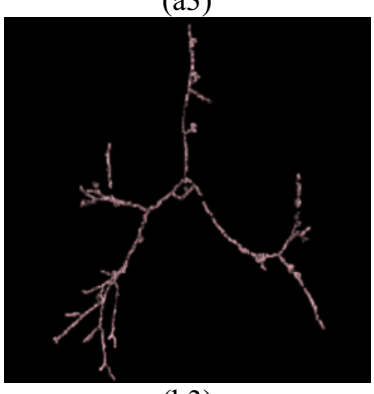

(b3)

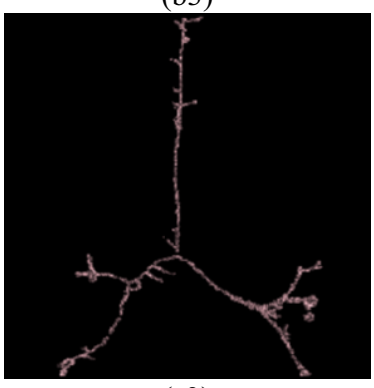

(c3)

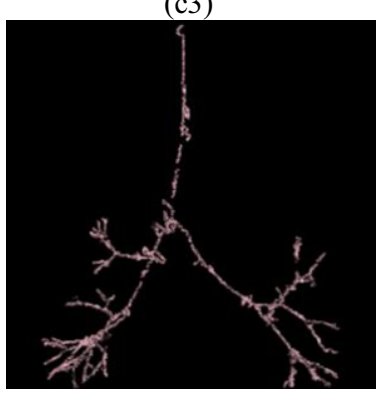

(d3)

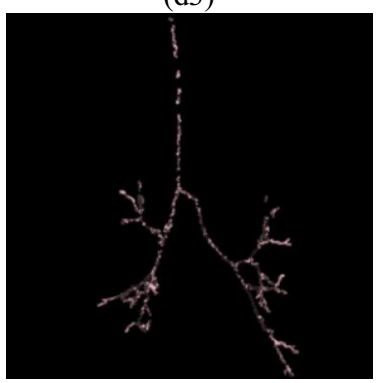

(e3)

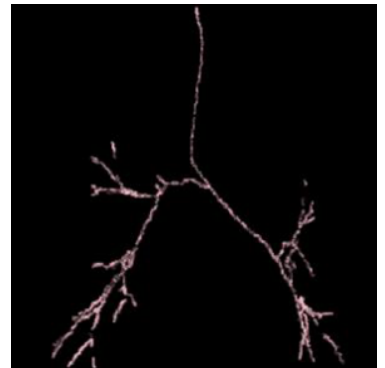

(a4)

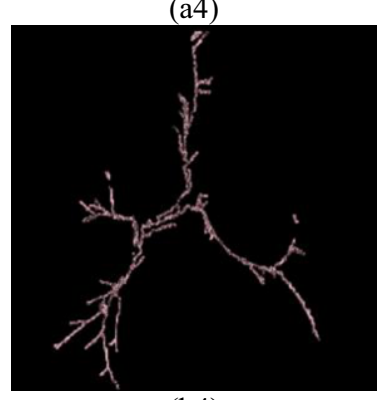

(b4)

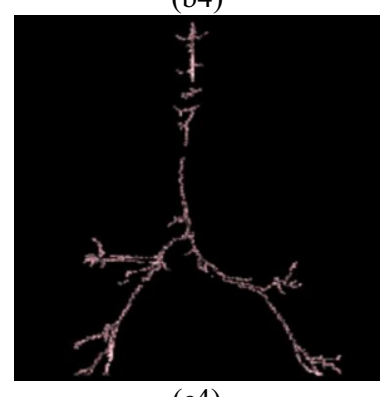

(c4)

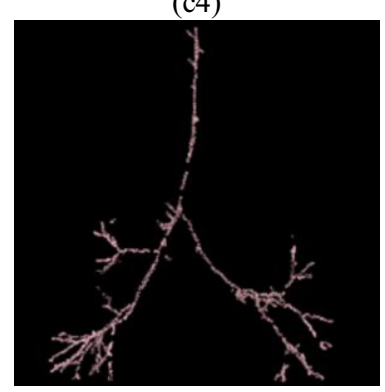

(d4)

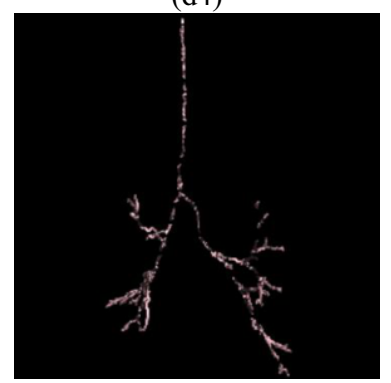

(e4) 


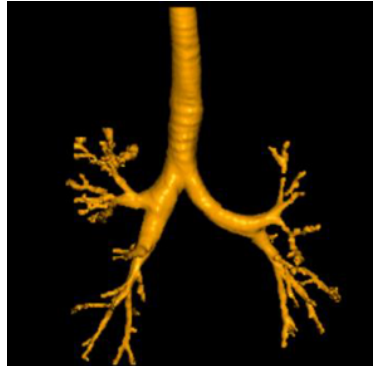

(f1)

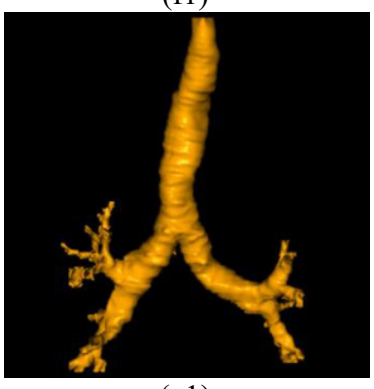

(g1)

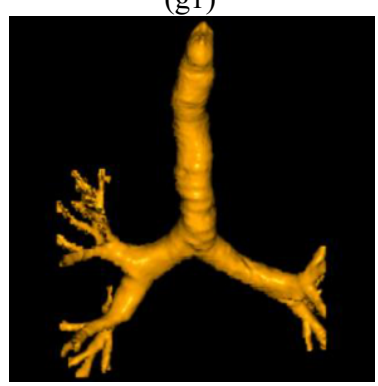

(h1)

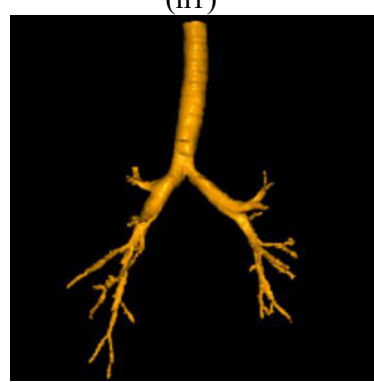

(i1)

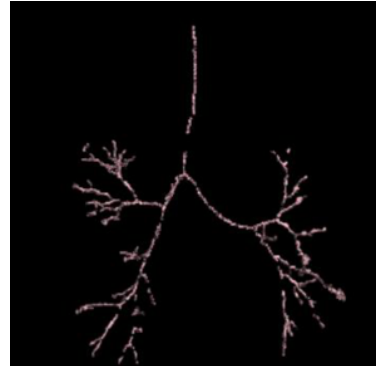

(f2)

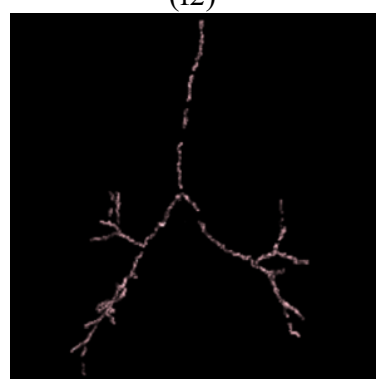

(g2)

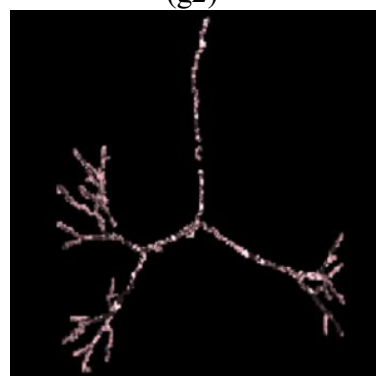

(h2)

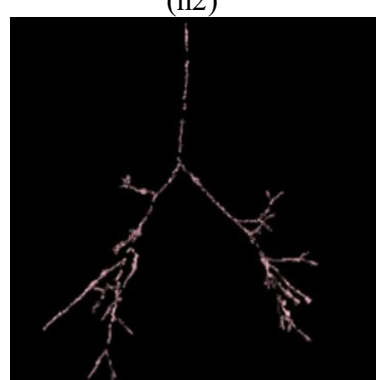

(i2)

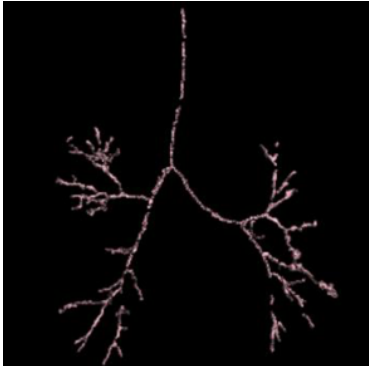

(f3)

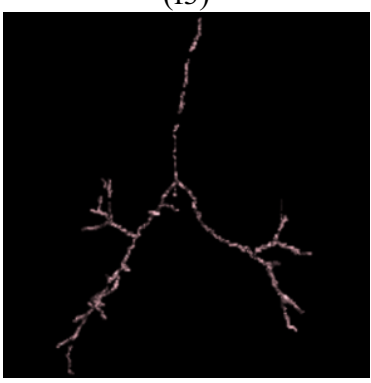

(g3)

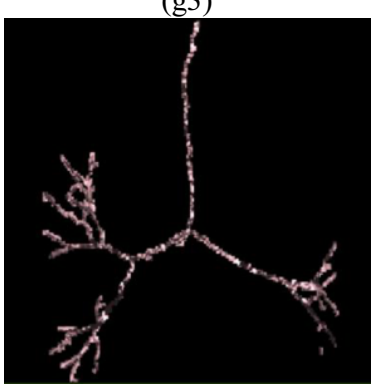

(h3)

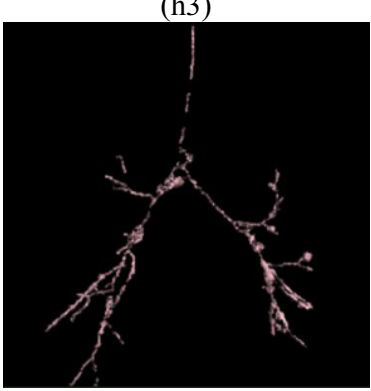

(i3)

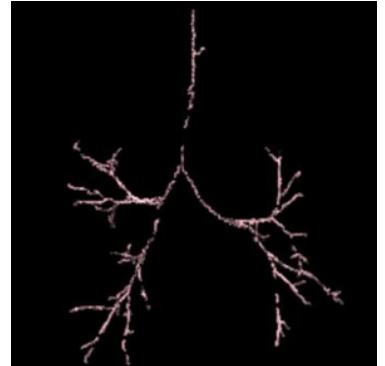

(f4)

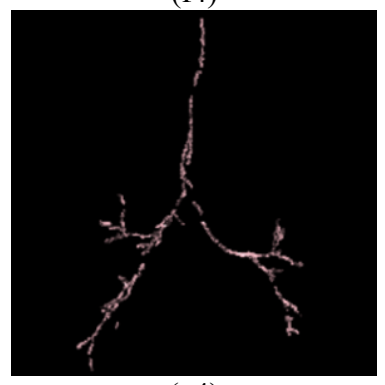

(g4)

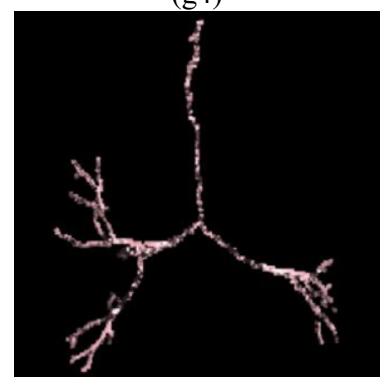

(h4)

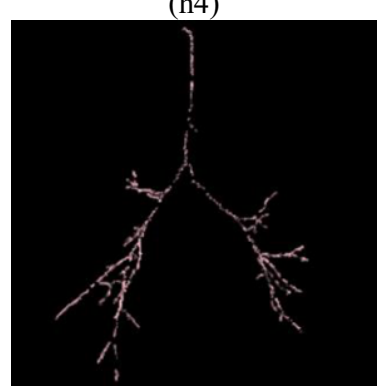

(i4)

Fig. 1. Segmented airway trees and their centerlines. (a1-i1) Airway (yellow), (a2-i2) proposed method-based, (a3-i3) thinning-based and (a4-i4) distance-based results (white). All nine case results are presented sequentially. 


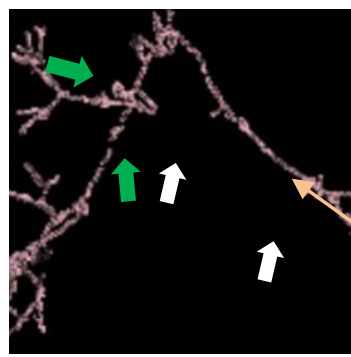

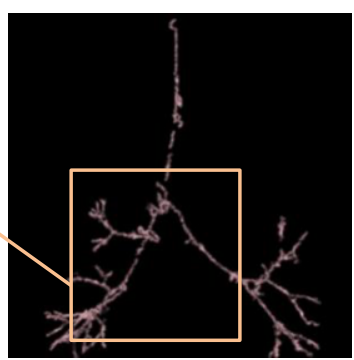

(a)

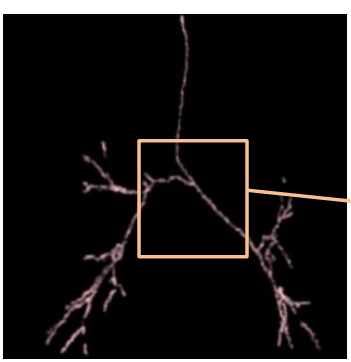

(b)

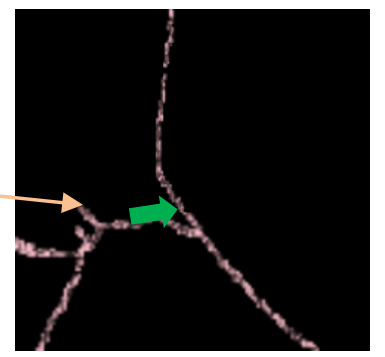

Fig. 2. False extraction: (a) Extra branches (green arrow pointed) and circles (white arrow pointed) in Case 4; (b) Centerline position bias (green arrow pointed) in Case 1 .

Table 3 provides different results by the multi-scale pruning strategy. It is obvious that the proposed method attains tendency towards stability when the threshold was 4: more than $96 \%$ branches were extracted in average, of which standard deviation was $1.9 \%$. On the contrary, there were great differences when the multi-scale strategy was utilized on traditional methods. The best result of extraction percentage was 7 and 9, respectively, whereas the standard deviations were as unacceptable as $6.0 \%$ and $7.7 \%$. The extraction results converged when the threshold was 11 with considerable low averages, $87.1 \%$ and $87.9 \%$, which indicates significant invalidation of the centerline generation procedure.

The proposed method statistically significantly outperformed traditional methods in the pruning test. Such advantage is beneficial from the application of prior anatomic knowledge. The proposed method recognizes false branches referred to not only their length but also their spatial structure. A number of normal short segments were preserved after the fitting step and avoided the varied refinement results.

Table 4 offers the comparison results of extracted centerlines after the filling phase with initial and

Table 3

Comparison of extraction results by the multi-scale strategy

\begin{tabular}{llllllll}
\hline & Thresholds & \multicolumn{7}{l}{} & & & \\
\cline { 2 - 7 } & 2 & 3 & 4 & 5 & 7 & 9 & 11 \\
\hline $\begin{array}{l}\text { The proposed } \\
\text { method }\end{array}$ & $106.5 \pm 15.0$ & $100.1 \pm 3.7$ & $\mathbf{9 6 . 3} \pm \mathbf{1 . 9}$ & $96.3 \pm 1.9$ & $96.3 \pm 1.9$ & $96.3 \pm 1.9$ & $96.3 \pm 1.9$ \\
$\begin{array}{l}\text { Thinning-based } \\
\text { method }\end{array}$ & $107.9 \pm 13.4$ & $104.1 \pm 11.5$ & $102.0 \pm 10.2$ & $97.0 \pm 8.2$ & $\mathbf{9 3 . 9} \pm \mathbf{6 . 0}$ & $90.9 \pm 4.7$ & $87.1 \pm 4.5$ \\
$\begin{array}{l}\text { Distance-based } \\
\text { method }\end{array}$ & $108.9 \pm 18.6$ & $103.2 \pm 14.7$ & $98.3 \pm 12.9$ & $95.0 \pm 10.5$ & $93.3 \pm 9.5$ & $\mathbf{8 9 . 5} \pm 7.7$ & $83.7 \pm 5.3$ \\
\hline
\end{tabular}

Table 4

Comparison between centerline results with and without filling process

\begin{tabular}{lllllll}
\hline & \multicolumn{3}{l}{ With initial centerlines } & \multicolumn{3}{l}{ With segmented airway trees } \\
\cline { 2 - 6 } & Branch (\%) & Volume (\%) & Surface (\%) & Branch (\%) & Volume (\%) & Surface (\%) \\
\hline $\begin{array}{l}\text { The proposed } \\
\text { method }\end{array}$ & $\mathbf{1 0 0 . 5} \pm \mathbf{1 . 1}$ & $\mathbf{1 0 0 . 2} \pm \mathbf{0 . 2}$ & $\mathbf{1 0 0 . 5} \pm \mathbf{0 . 5}$ & $\mathbf{9 6 . 8} \pm \mathbf{1 . 9}$ & $\mathbf{9 5 . 1} \pm \mathbf{1 . 5}$ & $\mathbf{9 5 . 6} \pm \mathbf{1 . 5}$ \\
$\begin{array}{l}\text { Thinning-based } \\
\text { method }\end{array}$ & $96.8 \pm 2.5$ & $100.4 \pm 0.4$ & $100.5 \pm 0.4$ & $104.2 \pm 11.2$ & $95.5 \pm 1.7$ & $95.9 \pm 1.5$ \\
$\begin{array}{l}\text { Distance-based } \\
\text { method }\end{array}$ & $97.4 \pm 5.1$ & $100.7 \pm 2.0$ & $101.3 \pm 1.4$ & $105.5 \pm 15.4$ & $93.7 \pm 4.4$ & $93.1 \pm 5.0$ \\
\hline
\end{tabular}


segmented data. The extraction with and without the hole filling phase returns fairly identical centerlines with $100.5 \pm 1.1 \%, 100.2 \pm 0.2 \%, 100.5 \pm 0.5 \%$ for the three given indices. Some short branches were additionally identified and the centerline centrality was improved slightly after the process. The results compared with original airway tree remain a superior consistency. These parameters imply that intraluminal cavities rarely influence on the proposed method. In other words, since the method can reject circles induced by the incomplete segmentation, it can be believed free from cavities.

Unfortunately, this identical phenomenon is not guaranteed by traditional methods. It can be found that the branch amount by thinning-based method experienced apparent decrease as segmented airway trees were preprocessed from

Table 4. The reproduced volume and surface area by distance-based method increase as the centerline position is corrected.

\section{Conclusion}

The proposed method is accurate and robust to varied segmented airway results and free from surface noise and intraluminal cavities. The thinning phase achieves a remarkably centered region with relative low computation complexity. The graph-theoretic analysis phase guarantees a tree structure without extra branches and circles. When tested in airway cases with different segmentation conditions, the method's performance has incomparable advantages in topology and geometry preserving. All the quantitative analysis information has been believed satisfactory for the accuracy and robustness requirement in disease diagnosis and treatment. This technique will be effective and for both clinical and fundamental imaging investigations of the lung. Some research will be spread out in the future. Further surface smoothing will be implemented to avoid extra branches before the extraction. The method will be tested as more volumetric images are obtained. Parallel thinning phase will be implemented using CUDA technology to achieve considerable faster extraction speed. Additional anatomic information will be then used in the refinement phase to identify false branches in a more reliable way.

\section{Acknowledgement}

This research was partly supported by National Natural Science Foundation of China (NSFC) under Grant No. 61302012 and No. 61172002, the Fundamental Research Funds for the Central Universities under Grant N130418002 and N120518001, and Liaoning Natural Science Foundation under Grant No. 2013020021.

\section{References}

[1] B. Wang, L. Miao, H. Dong and Z. Zheng, The research of lung sound signals based on cepstrum analysis, in: Proceedings of 2012 International Conference on Biomedical Engineering and Biotechnology, M.I. Vai, ed., IEEE Computer Society Conference Publishing Services, California, 2012, pp. 934-938.

[2] H. Yu, S.X. Zhang, R.H. Wang, G.Q. Zhang and J.M. Tan, The feasibility of mapping dose distribution of 4DCT images with deformable image registration in lung, Bio-Medical Materials and Engineering 24 (2014), 145-153.

[3] K. Palágyi, E. Balogh, A. Kuba, C. Halmai, B. Erdőhelyi, E. Sorantin and K. Hausegger, A sequential 3D thinning algorithm and its medical applications, in: Information Processing in Medical Imaging, Springer ,Berlin Heidelberg, 2001, pp. 409-415. 
[4] K. Palágyi, N. Gábor and K. Péter, Topology preserving parallel 3D thinning algorithms, in: Digital Geometry Algorithms, Springer, Netherlands, 2012, pp. 165-188.

[5] N. Gagvani and D. Silver, Parameter-controlled volume thinning, Graphical Models and Image Processing, 61.3 (1997), 149-164.

[6] B. Liu, C.T. Alexandru, B.T.M. R. Jos, J.C. Gordon, W. David, Y. Po, D. Feng, C. Valeriu and C. Alessandro, Parallel centerline extraction on the GPU, Computers \& Graphics 41 (2014), 72-83.

[7] T. Schlathoelter, C. Lorenz, I.C. Carlsen, S. Renisch and T. Deschamps, Simultaneous segmentation and tree reconstruction of the airways for virtual bronchoscopy, Medical Imaging, International Society for Optics and Photonics 4684 (2002), 103-113.

[8] J. Tschirren, K. Palágyi, J.M. Reinhardt, E.A. Hoffman and M. Sonka, Segmentation, skeletonization, and branchpoint matching — a fully automated quantitative evaluation of human intrathoracic airway trees, in: Medical Image Computing and Computer-Assisted Intervention, Springer, Berlin Heidelberg, 2002, pp. 12-19.

[9] K. Palágyi, J. Tschirren, E.A. Hoffman and M. Sonka, Quantitative analysis of pulmonary airway tree structures, Computers in Biology and Medicine 36 (2006), 974-996.

[10] T.C. Lee, R.L. Kashyap and C.N. Chu, Building skeleton models via 3-D medial surface axis thinning algorithms, CVGIP: Graphical Models and Image Processing 56 (1994), 462-478.

[11] S. Ukil and J.M. Reinhardt, Anatomy-guided lung lobe segmentation in X-ray CT images, IEEE Transactions on Medical Imaging 28 (2009), 202-214.

[12] P.B. Noble, R.A. McLaughlin, A.R. West, S. Becker, J.J. Armstrong, P.K. McFawn, P.R. Eastwood, D.R. Hillman, D.D. Sampson and H.W. Mitchell, Distribution of airway narrowing responses across generations and at branching points, assessed in vitro by anatomical optical coherence tomography, Respir. Res. 9 (2010), 1-12.

[13] W. Tan, J. Yang, D. Zhao, S. Ma, L. Qu and J. Wang, A novel method for automated segmentation of airway tree, in: Proceedings of the 2012 Chinese Control and Decision Conference, G. Yang, ed., IEEE Industrial Electronics (IE) Chapter, Singapore, 2012, pp. 976-979.

[14] P. Lo, B.V. Ginneken, J.M. Reinhardt, T. Yavarna, P.A. De Jong, B. Irving and M.D. Bruijne, Extraction of airways from CT (EXACT'09), IEEE Transactions on Medical Imaging 31 (2012), 2093-2107.

[15] P. Soille, Morphological Image Analysis: Principles and Applications, Springer-Verlag, New York, 2003. 